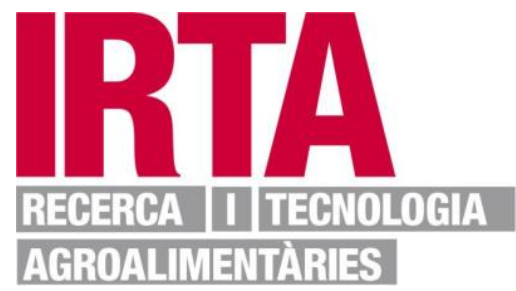

This document is a postprint version of an article published in Sensors and Actuators B: Chemical copyright $(\mathcal{O}$ Elsevier after peer review. To access the final edited and published work see $h$ ttps://doi.org/10.1016/.snb.2018.02.046 


\title{
Detection of azaspiracids in mussels using electrochemical immunosensors for fast screening in monitoring programs
}

Sandra Leonardo ${ }^{a}$, Jane Kilcoyne ${ }^{b}$, Ingunn A. Samdal' ${ }^{c}$ Christopher O. Miles ${ }^{c, d}$, Ciara K. O'Sullivan ${ }^{\mathrm{e}}{ }^{\mathrm{f}}$, Jorge Diogène ${ }^{\mathrm{a}}$, Mònica Campàs ${ }^{\mathrm{a} *}$

aIRTA, Ctra. Poble Nou, km. 5.5, 43540 Sant Carles de la Ràpita, Spain

${ }^{b}$ Marine Institute, Rinville, Oranmore, County Galway H91 R673, Ireland

'Norwegian Veterinary Institute, P.O. Box 750 Sentrum, N-0106 Oslo, Norway

${ }^{d}$ National Research Council Canada, 1411 Oxford St., Halifax, Nova Scotia, B3H 3Z1, Canada

eDepartament d'Enginyeria Química, Universitat Rovira i Virgili, Av. Països Catalans, 26, 43007

Tarragona, Spain

fInstitució Catalana de Recerca i Estudis Avançats, Pg. Lluís Companys, 23, 08010 Barcelona, Spain

*Corresponding author: Mònica Campàs

Tel.: +34 902789449 (ext. 1842); Fax: +34 977744138

E-mail address: monica.campas@irta.cat

\begin{abstract}
Given the widespread occurrence of azaspiracids (AZAs) it is clearly necessary to advance in simple and low-cost methods for the rapid detection of these marine toxins in order to protect seafood consumers. To address this need, electrochemical immunosensors for the detection of AZAs based on a competitive direct immunoassay using peroxidase-labelled AZA as a tracer were developed. An anti-AZA polyclonal antibody was immobilised in a controlled and stable manner on protein $\mathrm{G}$ or avidin-coated electrodes. Experimental conditions were first optimised using colorimetric immunoassays on microtitre plates, providing intermediate products already applicable to the accurate detection of AZAs. Then, transfer of the protein $\mathrm{G}$ and avidin-biotin interaction-based immunoassays to 8-electrode arrays provided compact and miniaturised devices for the high-throughput detection of AZAs. The low amounts of immunoreagents required as well as the potential for reusability of the avidin-biotin interaction-based immunosensors represented significant economic savings as well as a contribution to sustainability. The electrochemical immunosensors enabled the quantification of all regulated
\end{abstract}


AZAs below the regulatory limit, as well as a broad range of other toxic AZA analogues (from 63 \pm 3 to $2841 \pm 247 \mu \mathrm{g}$ AZA-1 equiv./kg for the protein G-based immunosensor and from $46 \pm 2$ to $3079 \pm 358 \mu \mathrm{g}$ AZA-1 equiv./kg for the avidin-biotin interaction-based immunosensor). The good agreement between the results obtained by the immunosensors and LC-MS/MS in the analysis of naturally contaminated mussel samples demonstrated the easy implementation of electrochemical immunosensors for routine analysis of AZAs in food safety monitoring programs.

Keywords: azaspiracid; antibody; electrochemical immunosensor; protein G; biotin; mussel. 


\section{INTRODUCTION}

Harmful algal blooms (HABs) and their associated marine toxins pose a serious threat to human health and are an economic concern for the shellfish industry. Among many different groups of marine toxins of microalgal origin are azaspiracids (AZAs). AZAs were first identified in 1998 from contaminated mussels from Ireland that had caused a poisoning outbreak in The Netherlands in the mid-1990s [1]. Since then, AZAs have been particularly problematic in Ireland, but they have also been reported in phytoplankton and/or shellfish around the world including the US [2], China [3], Japan [4], Chile [5, 6] and Argentina [7]. In Europe, AZAs have been found in several countries adjacent to the North Sea such as Norway, Denmark, UK and Sweden [8-10]. AZAs have also been found in the Atlantic coast of other European countries including France, Portugal and Spain [11-15], in the Atlantic coast of NW Africa [16], as well as in countries of the Mediterranean Sea [17].

The AZA group includes more than 40 analogues, which are either produced by phytoplankton of the genera Azadinium and Amphidoma, through biotransformation in shellfish, or as byproducts resulting from storage or cooking of AZA-contaminated shellfish [18]. However, only AZA-1-3 are currently regulated by the European Commission, with $160 \mu \mathrm{g}$ AZA-1 equivalents $/ \mathrm{kg}$ being the maximum permitted level in shellfish meat [19].

Although current reference chemical methods are highly specific and sensitive, they require the use of sophisticated equipment and trained personnel, being expensive and relatively slow to perform. Thus, there is a need for easy-to-use, rapid, inexpensive and accurate devices for the detection of AZAs in shellfish in monitoring programs. Biosensors have the potential to address this need and, among them, electrochemical biosensors stand out for several reasons: their inherently high sensitivities, low cost, possibility for miniaturisation of electrodes and potentiostats, compatibility with microfluidics systems and automation and subsequent simplification of the protocols [20]. Likewise, the high specificity and selectivity of antibodies (Abs) positions immunosensors as highly attractive candidates for the rapid screening of marine toxins.

Nonetheless, the lack of commercially available anti-AZA Abs has hindered the development of immunosensors, as well as immunoassays, for the detection of AZAs. To date, only a monoclonal antibody (MAb) raised using AZA-1 [21] and a polyclonal antibody (PAb) raised using a synthetic fragment of AZA [22] have been reported. While the MAb was used in the development of a flow fluorimetry-based immunoassay [23], the anti-AZA PAb has been used in the development 
of an enzyme-linked immunosorbent assay (ELISA) [24] and an electrochemical immunoassay using magnetic beads as immunorecognition supports [25].

Another crucial step in the development of immunoassays and immunosensors is the immobilisation of the Ab on a solid support. To achieve full functionality, the conformation of the Abs should not be altered and their binding sites should remain accessible after immobilisation. Moreover, in the development of electrochemical biosensors, the immobilisation procedure should ensure a close proximity between the label and the transducer in order to obtain an efficient electron transfer. Bioaffinity immobilisation, mainly based on the avidin-biotin interaction and the affinity of protein A/G for immunoglobulins (IgGs), provides an attractive method for the controlled and stable surface-tethering of antibodies. In our previous work [25], the anti-AZA PAb was immobilised on protein G-coated magnetic beads and a competitive step using peroxidase-labelled AZA (AZA-HRP) was performed. The use of magnetic beads facilitated performance of the assay in suspension, thus allowing rapid assay kinetics, but mass transfer limitations were observed when the immunocomplexes were subsequently anchored on the electrode surface to perform electrochemical detection. In the present work, the anti-AZA PAb is immobilised on the electrode surface by means of protein $G$, or, alternatively, through the avidin-biotin interaction, with the aim of overcoming the drawbacks associated with the packed distribution of magnetic beads on the electrode surface and allowing a controlled and homogenous immobilisation of the antibody directly on the transducer surface. The immobilisation of the Ab directly on the transducer surface provides more compact and automated devices, since all reactions are performed on the electrode array, with possible signal enhancement since the enzyme product is concentrated closer to the transducer surface.

Colorimetric immunoassays were first developed on microtitre plates and used for protocol optimisation and assay characterisation. Their suitability for AZAs screening and quantification in mussels was also demonstrated. Protein $\mathrm{G}$ and avidin-biotin interaction-based immunoassays were then transferred to electrode arrays to develop the corresponding competitive electrochemical immunosensors. With the aim of further improving the economic saving represented by the use of the immunosensors, their reusability was explored. The immunosensors were applied to the determination of AZAs in a mussel certified reference material (CRM) and in mussel samples obtained from the Irish monitoring program. To the best of our knowledge, this work describes the first immunosensors for AZAs detection and guarantees their implementation in routine monitoring programs. 


\section{MATERIALS AND METHODS}

\subsection{Reagents and solutions}

Protein G from Streptococcus sp., avidin from egg white, sodium carbonate, sodium bicarbonate, potassium phosphate monobasic, potassium chloride, Tween-20, bovine serum albumin (BSA) and 3,3'-5,5'-tetramethylbenzidine (TMB) liquid substrate were purchased from Sigma-Aldrich (Madrid, Spain). Milli-Q water (Millipore, Bedford, USA) was used to prepare solutions.

Certified reference materials (CRMs) of AZA-1-3, Zero-Mus and AZA-Mus were obtained from the National Research Council of Canada (NRC, Halifax, NS, Canada). Reference materials (RMs) of AZA-4-10 were prepared as described by Kilcoyne et al. [26].

HRP (Type VI-A) from Sigma-Aldrich (Oslo, Norway) was conjugated to purified AZA-1 [27] using standard procedures for covalent linking of small molecules to enzymes [28]. Antiserum AgR36711b (anti-AZA PAb) was obtained after 11 immunisations with two different haptens as described in Samdal et al. [24].

Biotin labelling of the anti-AZA PAb was performed with EZ-Link ${ }^{\mathrm{TM}}$ NHS-PEG ${ }_{4}$-Biotin from Thermo Fisher (Barcelona, Spain) following the manufacturer's manual. Unreacted NHS-PEG Biotin was removed by Zeba Spin Desalting Colums (7 kDa MWCO, $2 \mathrm{~mL}$ ) from Thermo Fisher.

\subsection{Equipment}

Colorimetric measurements were performed with a Microplate Reader KC4 from BIO-TEK Instruments, Inc. (Winooski, VT, USA). Gen5 software was used to collect and evaluate data. An array of eight screen-printed carbon electrodes (DRP-8X110) and a boxed connector (DRPCAST8X) were provided by Dropsens S.L. (Oviedo, Spain). The array consists of 8 carbon working electrodes of $2.5 \mathrm{~mm}$ in diameter, each with its own carbon counter electrode and silver reference electrode. Amperometric measurements were performed with a PalmSens potentiostat connected to an 8-channel multiplexer (MUX8) (Houte, The Netherlands). Data were collected and evaluated with PalmSens PC software.

\subsection{Raw and heat-treated mussel tissues}

AZA-Mus CRM (NRC, Halifax, NS, Canada) prepared from naturally contaminated mussel (Mytilus edulis) tissues and AZA-contaminated raw mussel samples ( $M$. edulis) from the routine 
monitoring program in Ireland, were selected for analysis. Zero-Mus CRM (M. edulis) (NRC, Halifax, NS, Canada) was used to evaluate matrix effects.

Mussels were shucked and homogenised with an Ultra Turrax homogenizer. A three-step extraction with $\mathrm{MeOH}(10 \mathrm{~mL})$ was performed on AZA-Mus and Zero-Mus homogenised tissues (1 g) according to Gerssen et al. [29], using a protocol that was intra-laboratory validated by García-Altares et al. [30]. A vortex-mixer MS2 Minishaker (IKA Labortechnik, Staufen, Germany) and a centrifuge Jouan MR 23i (Thermo Fisher Scientific Inc., Waltham, MA, USA) were used. Crude extracts were filtered through polytetrafluoroethylene (PTFE) 0.2- $\mu \mathrm{m}$ membrane syringe filters. Thus, AZA-Mus and Zero-Mus extracts at a matrix concentration of $100 \mathrm{mg} / \mathrm{mL}$ were obtained.

Extraction of the Irish AZA-contaminated raw mussel samples was performed by a two-step extraction with $\mathrm{MeOH}(25 \mathrm{~mL})$ as follows. Homogenised tissue samples were weighed $(2 \mathrm{~g})$ into 50-mL centrifuge tubes and extracted by vortex mixing for 1 min with $9 \mathrm{~mL}$ of $\mathrm{MeOH}$, centrifuged at $3950 \mathrm{~g}(5 \mathrm{~min})$, and the supernatants decanted into $25-\mathrm{mL}$ volumetric flasks. The pellets were further extracted using an Ultra Turrax for 1 min with an additional $9 \mathrm{~mL}$ of $\mathrm{MeOH}$, centrifuged at $3950 \mathrm{~g}(5 \mathrm{~min})$, and the supernatants decanted into the same $25-\mathrm{mL}$ volumetric flasks, which were brought to volume with $\mathrm{MeOH}$. A portion $(10 \mathrm{~mL})$ of each extract was transferred into sealed centrifuge tubes and placed for $10 \mathrm{~min}$ in a water bath heated to $90^{\circ} \mathrm{C}$ to decarboxylate carboxylated AZAs [31, 32]. The raw and heat-treated samples were then passed through Whatman 0.2- $\mu \mathrm{m}$ cellulose acetate filters into HPLC vials for analysis. Heated and raw mussel extracts were obtained at a matrix concentration of $80 \mathrm{mg} / \mathrm{mL}$. All samples were stored at -20 ${ }^{\circ} \mathrm{C}$ until analysis.

\subsection{Colorimetric immunoassays protocol}

Colorimetric immunoassays were carried out on 96-well microtitre plates. Microtitre wells were incubated with $50 \mu \mathrm{L}$ of $10 \mu \mathrm{g} / \mathrm{mL}$ protein $\mathrm{G}$ or $1 \mu \mathrm{g} / \mathrm{mL}$ avidin in $0.1 \mathrm{M}$ carbonate buffer, $\mathrm{pH}$ 9.6, for $1 \mathrm{~h}$. The anti-AZA PAb or biotinylated PAb was then immobilised on the protein $\mathrm{G}$ or avidincoated plates, respectively, by the addition of $50 \mu \mathrm{L}$ of the corresponding antibody dilution (from $1 / 10000$ to $1 / 80000$ for protocol optimisation and $1 / 40000$ for the final competition assay) in PBS-Tween (0.1 M PBS, pH 7.2, with 0.05\% v/v Tween-20) for $1 \mathrm{~h}$. Blocking was then carried out using $100 \mu \mathrm{L}$ of PBS-Tween containing $2 \% \mathrm{w} / \mathrm{v}$ BSA for $1 \mathrm{~h}$. Subsequently, the competition step was performed using $25 \mu \mathrm{L}$ of AZA-1 standard solutions (from $0.20 \mu \mathrm{g} / \mathrm{L}$ to $100 \mu \mathrm{g} / \mathrm{L}$ ) or natural samples at different dilutions in PBS-Tween and $25 \mu \mathrm{L}$ of different dilutions of AZA-HRP (from 
$1 / 800$ to $1 / 3200$ for protocol optimisation and $1 / 1600$ for the final competition assay) for 30 min. Finally, $100 \mu \mathrm{L}$ of TMB liquid substrate was added, and 10 min later the absorbance was read at $620 \mathrm{~nm}$. After each step, wells were rinsed three times with $100 \mu \mathrm{L}$ PBS-Tween. During incubations, microtitre plates were placed on a plate shaker. All reactions were carried out at room temperature.

\subsection{Electrochemical immunosensors protocol}

The immunosensor assay protocols were essentially the same as the colorimetric immunoassays except for adjustments to the volumes for 8-electrode arrays and diffences in the detection step. Volumes of $10 \mu \mathrm{L}$ were applied to each working electrode $(5 \mu \mathrm{L}$ of standard or sample dilution plus $5 \mu \mathrm{L}$ of AZA-HRP in the competition step), and the blocking step was performed by immersion of the electrode arrays in PBS-Tween containing $2 \% \mathrm{w} / \mathrm{v}$ BSA. To perform the electrochemical measurement, $10 \mu \mathrm{L}$ of TMB was added to each electrode and incubated for 10 min and, finally, the TMB reduction current was measured by applying $-0.2 \mathrm{~V} v$ s. Ag for $0.5 \mathrm{~s}$. After each step, the electrode arrays were rinsed with PBS-Tween and dried. All reactions were carried out at room temperature.

For the regeneration of the electrochemical immunosensors, the electrode arrays were rinsed with PBS-Tween after the electrochemical measurement and immersed in glycine buffer, $\mathrm{pH}$ 2.7, for $30 \mathrm{~min}$. The immunosensors were then rinsed again with PBS-Tween and stored at $4{ }^{\circ} \mathrm{C}$ until use.

\subsection{LC-MS/MS analysis}

For LC-MS/MS analysis of AZA analogues, a Waters Acquity UPLC coupled to a Xevo G2-S QToF monitoring in $\mathrm{MS}^{\mathrm{e}}$ mode $(\mathrm{m} / \mathrm{z} 100-1200)$ was used with leucine enkephalin as the reference compound. The cone voltage was $40 \mathrm{~V}$, collision energy was $50 \mathrm{~V}$, the cone and desolvation gas flows were set at 100 and $1000 \mathrm{~L} / \mathrm{h}$, respectively, and the source temperature was $120{ }^{\circ} \mathrm{C}$. Analytical separation was performed on an Acquity UPLC BEH C18 $(50 \times 2.1 \mathrm{~mm}, 1.7 \mu \mathrm{m})$ column (Waters). Binary gradient elution was used, with phase $A$ consisting of $\mathrm{H}_{2} \mathrm{O}$ and phase $\mathrm{B}$ of $\mathrm{CH}_{3} \mathrm{CN}$ (95\%) in $\mathrm{H}_{2} \mathrm{O}$ (both containing $2 \mathrm{mM}$ ammonium formate and $50 \mathrm{mM}$ formic acid). The gradient was from $30-90 \%$ B over $5 \mathrm{~min}$ at $0.3 \mathrm{~mL} / \mathrm{min}$, held for $0.5 \mathrm{~min}$, and returned to the initial conditions and held for $1 \mathrm{~min}$ to equilibrate the system. The injection volume was $2 \mu \mathrm{L}$ and the column and sample temperatures were $25^{\circ} \mathrm{C}$ and $6{ }^{\circ} \mathrm{C}$, respectively. AZA-1-3 were quantified relative to CRMs while AZA-4-10 were quantified with RMs [26]. Matrix interferences were 
assessed using single point matrix matched standards for AZA-1-6 and AZA-8, using a blank $M$. edulis tissue. An aliquot ( $2 \mathrm{~g}$ ) of tissue was extracted as described above, this time making the solution up to a final volume of $20 \mathrm{~mL}$. The matrix-matched standard was prepared by adding $1.25 \mathrm{~mL}$ of an AZA-1-6 and AZA-8 stock solution in $\mathrm{MeOH}$ to $1 \mathrm{~mL}$ of the filtered (Whatmann, $0.2 \mu \mathrm{m}$, cellulose acetate filter) blank tissue extract. In parallel, a $\mathrm{MeOH}$ standard was prepared by adding $1.25 \mathrm{~mL}$ of the same AZA-1-6 and AZA-8 stock solution to $1 \mathrm{~mL}$ of $\mathrm{MeOH}$.

\subsection{Data analysis}

Measurements were performed in triplicate for the colorimetric immunoassays and electrochemical immunosensors and in duplicate for LC-MS/MS analysis. The immunoassay calibration curves were fitted using a sigmoidal logistic four-parameter equation. Linear regression was used to evaluate the correlation between AZA-1 equivalent concentrations in naturally contaminated mussel samples determined with the colorimetric immunoassays or the electrochemical immunosensors and the values obtained from the LC-MS/MS analyses. To evaluate differences between approaches, data were first tested for normality. To compare values from two different groups, the paired $t$-test was used for normally distributed data sets, while Wilcoxon matched-pairs signed-ranks was used for non-normally distributed data. Oneway ANOVA was performed to compare the values obtained in the analysis of the mussel samples by the colorimetric immunoassays, electrochemical immunosensors and LC-MS/MS analysis. Differences were considered statistically significant at the 0.05 level. SigmaStat 3.1 was used for statistical analysis. 


\section{RESULTS AND DISCUSSION}

\subsection{Colorimetric immunoassays}

Microtitre plates were coated with protein $\mathrm{G}$ or avidin by passive adsorption, taking advantage of the hydrophobic interactions and the electrostatic forces generated between the negatively charged proteins and the positively charged microplates in alkaline conditions. The anti-AZA PAb was then immobilised on the coated plates by means of the affinity of the protein $\mathrm{G}$ to the Fc region of the antibody or through the strong avidin-biotin interaction following antibody biotinylation (Fig. 1). Both bioaffinity interactions provided the stable immobilisation of the PAb, while retaining its biological activity. However, while the use of protein $\mathrm{G}$ favoured the optimum orientation of the antibody to achieve optimal antigen binding without requiring any chemical modification, the avidin-biotin interaction required biotin labelling of the primary amines of the PAb, typically distributed on the exterior of the entire antibody, which did not ensure the correct orientation of the antibody.

a

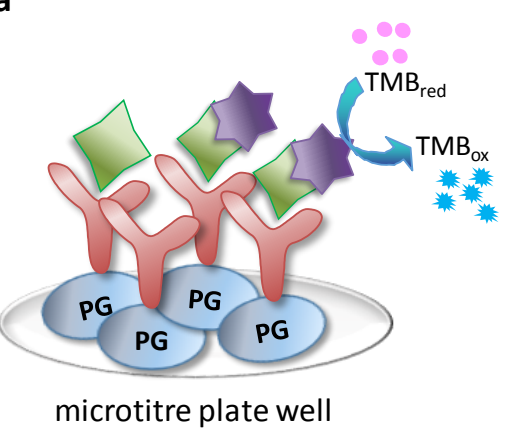

$[A b s]=620 \mathrm{~nm}$

b

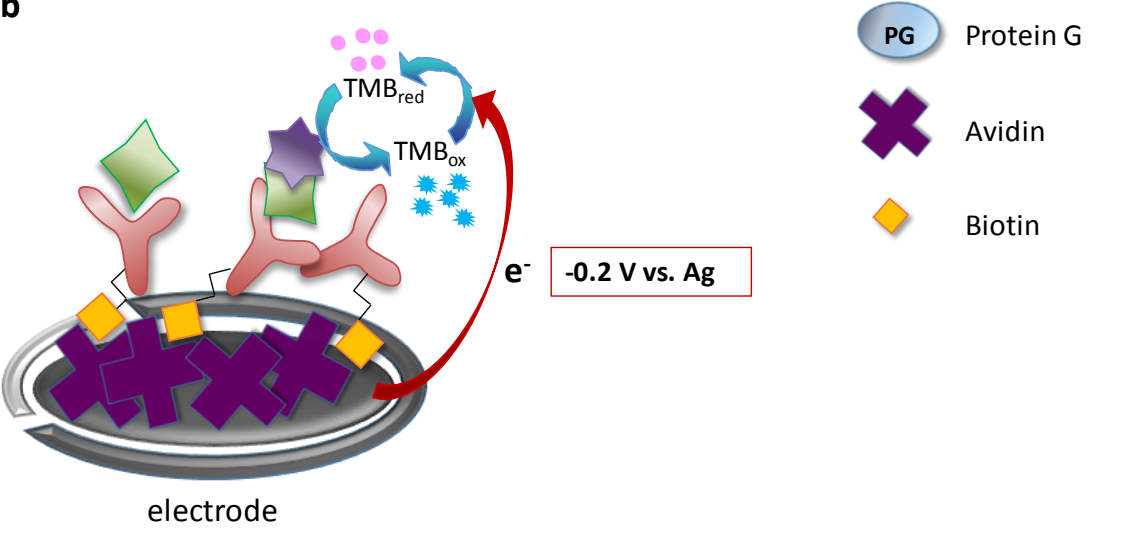

Fig. 1. Schematic representation of the immunoassays and immunosensors configurations achieved by the anti-AZA PAb immobilisation based on (a) protein $\mathrm{G}$ or (b) avidin-biotin affinity interaction on (a) microtitre plate wells and (b) electrodes, as examples. 
Checkerboard titrations and competitive immunoassays were performed to determine if the antibody immobilisation was successful and to establish the optimum PAb and AZA-HRP concentrations. The absorbance values obtained showed expected trends according to antibody and AZA-HRP tracer concentrations. Lower absorbance values were obtained when the antibody was immobilised through the avidin-biotin interaction. Increasing the amount of avidin on the plate did not improve the immobilisation yield of the biotinylated antibody. Nevertheless, the lower absorbance values achieved in this configuration are not unexpected taking into account the biotinylation yield, which may not reach $100 \%$, the non-optimally oriented immobilisation of the PAb and/or the possibility of biotinylation and immobilisation of other molecules containing primary amines present in the antiserum.

Both immobilisation strategies provided very low non-specific adsorption values of the AZAHRP. Calibration curves demonstrated competition of free AZA-1 with AZA-HRP for PAb binding. Calibration curves were background-corrected with respect to the controls with no AZA-HRP and fitted to the sigmoidal logistic four-parameter equation:

$$
y=y_{0}+\frac{a}{1+\left(\frac{x}{x_{0}}\right)^{b}}
$$

where $a$ and $y_{0}$ are the asymptotic maximum and minimum values, respectively, $x_{0}$ is the $x$ value at the inflection point and $b$ is the slope at the inflection point. Greater sensitivities were achieved with decreasing concentrations of antibody and tracer. Consequently, 1/40000 PAb and 1/1600 AZA-HRP dilutions were selected as a compromise between low antibody/tracer loading and sufficiently high absorbance values. Figure 2 shows the calibration curves for the optimised protein $G$ and avidin-biotin interaction-based immunoassays. In table 1, limits of detection (LODs), established as the $10 \%$ inhibition coefficient $\left(\mathrm{IC}_{10}\right)$ and working ranges (between $\mathrm{IC}_{20}$ and $\mathrm{IC}_{80}$ ) are presented together with the equations and the corresponding $R^{2}$ values. Differences between the two approaches were not significant $(t=0.292, P=0.774)$. In comparison with the competitive colorimetric immunoassay previously reported by our group [25], where magnetic beads were used as antibody immobilisation supports, it was possible to use lower antiserum and tracer concentrations, which could explain the lower LODs achieved in these approaches. 


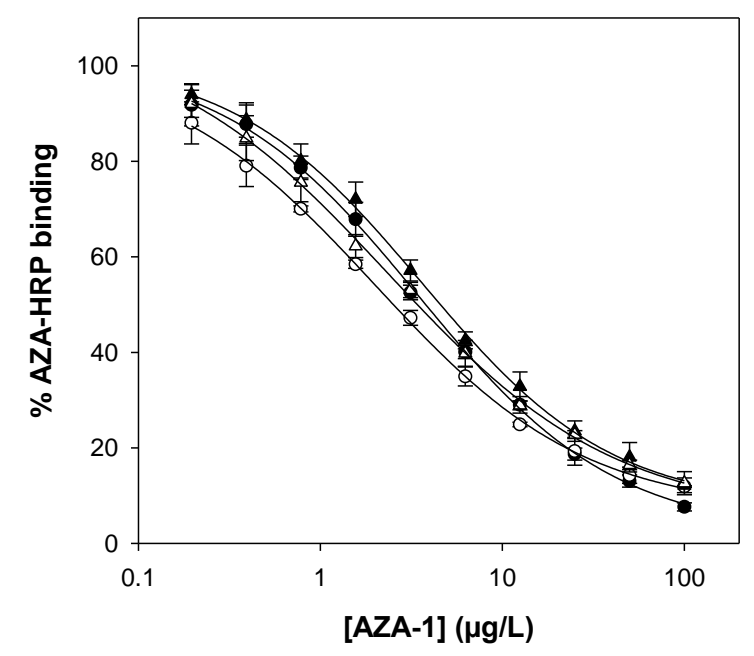

Fig. 2. Colorimetric calibration curves for AZA-1 obtained using the protein G-based immunoassay (black) and the avidin-biotin interaction-based immunoassay (white) in buffer (circle) and in $20 \mathrm{mg} / \mathrm{mL}$ mussel matrix with $20 \% \mathrm{MeOH}$ (triangle).

Table 1. Analytical parameters derived from the sigmoidal logistic four-parameter fitting for the Protein G-based and avidin-biotin interaction-based colorimetric immunoassays and electrochemical immunosensors.

\begin{tabular}{|c|c|c|c|c|c|}
\hline & & $\begin{array}{c}\operatorname{LOD}\left(\mathrm{IC}_{10}\right) \\
(\mu \mathrm{g} / \mathrm{L})\end{array}$ & $\begin{array}{l}\text { Working range } \\
\left(\mathrm{IC}_{20}-\mathrm{IC}_{80}\right)(\mu \mathrm{g} / \mathrm{L})\end{array}$ & Equation & $R^{2}$ \\
\hline \multirow{2}{*}{ 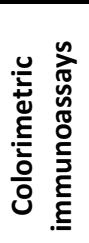 } & Protein G & 0.28 & $0.70-22.74$ & $y=2.052+\frac{99.494}{1+\left(\frac{x}{3.468}\right)^{0.805}}$ & 0.999 \\
\hline & Avidin-biotin & 0.14 & $0.39-22.47$ & $y=6.776+\frac{94.670}{1+\left(\frac{x}{1.999}\right)^{0.751}}$ & 1.000 \\
\hline \multirow{2}{*}{ 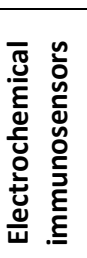 } & Protein G & 0.61 & $1.25-56.81$ & $y=-10.073+\frac{132.192}{1+\left(\frac{x}{5.413}\right)^{0.520}}$ & 0.999 \\
\hline & Avidin-biotin & 0.37 & $0.92-61.58$ & $y=-74.378+\frac{208.612}{1+\left(\frac{x}{32.208}\right)^{0.295}}$ & 0.999 \\
\hline
\end{tabular}

\subsection{Study of matrix effects}

Prior to the analysis of mussel samples, AZA-1 calibration curves using a blank certified reference mussel tissue matrix (CRM-Zero-Mus) were performed to evaluate matrix effects on the immunoassays. A matrix concentration of $20 \mathrm{mg} / \mathrm{mL}$ mussel matrix was chosen according to the 
protocol for lipophilic toxins extraction in shellfish (100 mg of matrix in $1 \mathrm{~mL}$ of $\mathrm{MeOH}$ ) and its subsequent dilution to $20 \% \mathrm{v} / \mathrm{v} \mathrm{MeOH}$, a percentage that had been previously demonstrated not to interfere with the assay [25]. No significant differences were observed between the calibration curves performed in buffer and in $20 \mathrm{mg} / \mathrm{mL}$ mussel matrix with $20 \% \mathrm{v} / \mathrm{v} \mathrm{MeOH}$, neither in the protein $\mathrm{G}$-based immunoassay $(\mathrm{t}=0.24, \mathrm{P}=0.81)$ nor in the immunoassay based on the avidin-biotin interaction ( $t=0.32, \mathrm{P}=0.77$ ) (Fig. 2). Consequently, considering a $20 \mathrm{mg} / \mathrm{mL}$ matrix loading, effective LODs of $14 \pm 1 \mu \mathrm{g}$ AZA-1 equiv./kg and $7 \pm 2 \mu \mathrm{g}$ AZA-1 equiv./kg were achieved for the immunoassays developed by means of protein $G$ and avidin-biotin immobilisation, respectively. Both immunoapproaches provided a broad working range, from $35 \pm 5 \mu \mathrm{g}$ AZA-1 equiv./kg to $1137 \pm 150 \mu \mathrm{g}$ AZA-1 equiv./kg in the case of the protein G-based immunoassay and from $20 \pm 3 \mu \mathrm{g}$ AZA-1 equiv./kg to $1124 \pm 118$ in the case of the immunoassay based on the avidin-biotin interaction. Considering the EC regulatory limit for AZAs of $160 \mu \mathrm{g} / \mathrm{kg}$, the immunoassays enabled the quantification of mussel samples from far below to far above the regulatory limit without requiring additional sample dilutions.

\subsection{Electrochemical immunosensors}

After protocol optimisation using the colorimetric immunoassays, both strategies were transferred to 8-electrode arrays to develop the corresponding electrochemical immunosensors (Fig. 1). Although reagent concentrations were the same as those selected in the colorimetric immunoassays, the use of lower volumes on the screen-printed electrodes required 5-fold lower amounts of protein G, avidin, anti-AZA PAb and tracer, which represents a substantial economic improvement. After background correction with respect to the controls with no AZA-HRP and fitting the calibration curves to the sigmoidal logistic four-parameter equation, Protein $\mathrm{G}$ and avidin-biotin interaction-based immunosensors provided similar analytical performances $(t=0.10, P=0.92)$ (Table 1, Fig. 3). This work presents the first immunosensors for AZAs reported to date, providing user-friendly and compact tools that favour operation with low sample volumes and the performance of multiple measurements in a short time.

Considering a 5 -fold sample dilution to $20 \mathrm{mg} / \mathrm{mL}$ mussel matrix, as in the colorimetric assays, effective working ranges between $63 \pm 3$ and $2841 \pm 247 \mu \mathrm{g}$ AZA-1 equiv./kg for the protein Gbased immunosensor and between $46 \pm 2$ and $3079 \pm 358 \mu \mathrm{g}$ AZA-1 equiv./kg for the avidinbiotin interaction-based immunosensor were calculated, again providing a broad working range that included the EC permitted threshold value. 


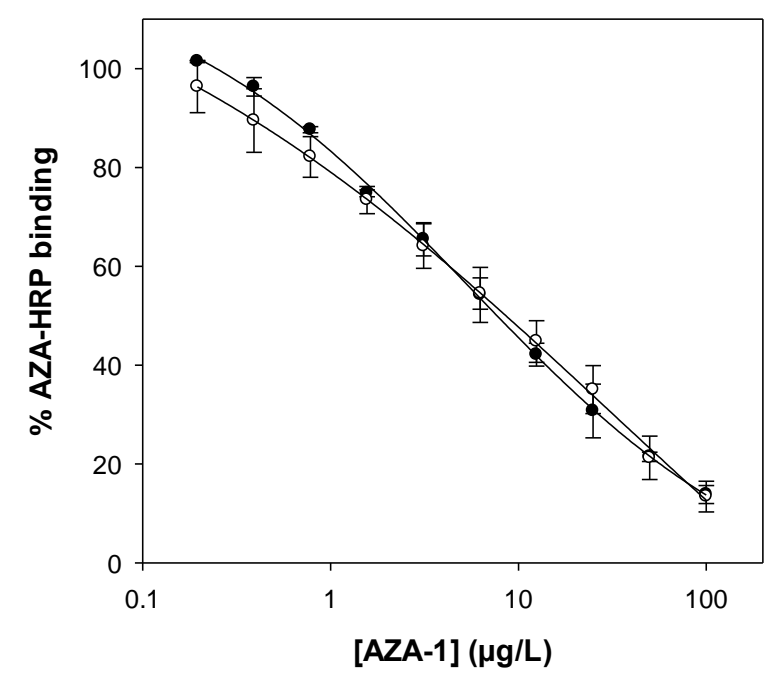

Fig. 3. Electrochemical calibration curves for AZA-1 obtained using the protein G-based immunosensor (black) and the avidin-biotin interaction-based immunosensor (white) in buffer.

\subsection{Regeneration of the electrochemical immunosensors}

Electrochemical immunosensors are commonly developed using disposable screen-printed electrodes due to their low fabrication cost and the possibility of mass production. However, the lack of commercially available anti-AZA antibodies and the time-consuming antibody production process are clear limiting factors in the development of immunosensors for AZAs detection. The feasibility to reuse immunosensors presents expedient advantages regarding these limitations. With this purpose in mind, the possibility to remove the AZAs and the AZA-HRP bound to the PAb, once the competition assay had been performed, while retaining the PAb immobilisation on the electrode surface and its functionality was evaluated.

Antibody-antigen interaction usually occurs at physiological $\mathrm{pH}$ and ionic strength, such as in PBS, and can be disrupted by simply raising or lowering the $\mathrm{pH}$ or altering the ionic state, ideally releasing the antibody or antigen without irreversibly denaturing or inactivating them. Thus, when immunosensors containing AZA-HRP were immersed in glycine buffer, $\mathrm{pH}$ 2.7, for 30 min, only background currents were observed after TMB incubation, indicative of complete AZA-HRP elution from the electrode. A subsequent incubation with AZA-HRP resulted in a response of $\sim 25 \%$ in the protein G-based functionalised electrodes, and a response of $\sim 100 \%$ in the avidinbiotin configuration. This $100 \%$ response demonstrates that the biotinylated antibody was retained on the avidin-coated electrode retaining its functionality, while the $25 \%$ response observed in the protein $\mathrm{G}$ approach suggests partial co-elution of the antibody during the elution 
step in this specific configuration. Responses close to $100 \%$ following AZA-HRP incubation were maintained after the regeneration of the functionalised electrodes through the avidin-biotin interaction for 6 consecutive times on 3 different days (Fig. 4). These results demonstrate the reusability of the avidin-biotin interaction-based immunosensors and also highlight the storage stability of the immunosensors for at least 3 days. The possibility to reuse and store the immunosensors until use avoids the immobilisation of additional anti-AZA PAb amounts on the electrode surface, as well as simplifies and shortens the protocol assay.

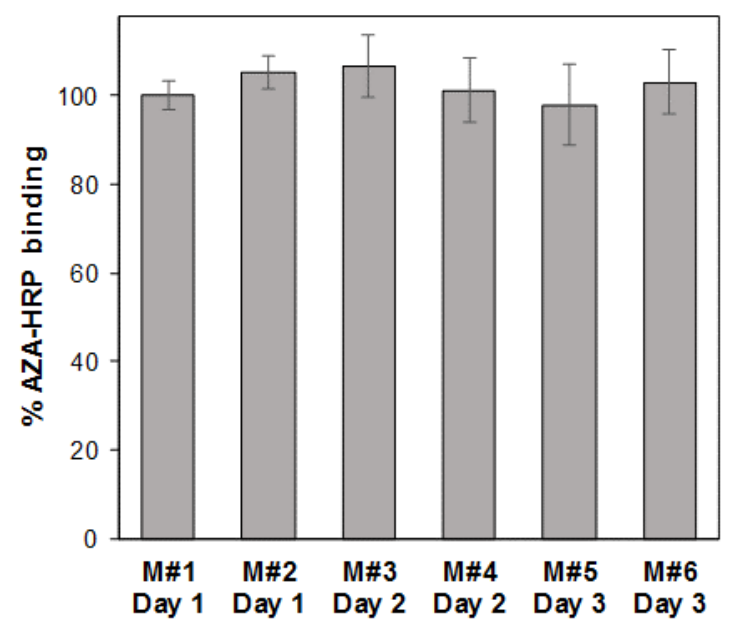

Fig. 4. Electrochemical responses of the avidin-biotin interaction-based immunosensors achieved after surface regeneration for 6 consecutive measurements (M\#1-M\#6) on 3 different days.

\subsection{AZAs detection in mussel samples}

To demonstrate the applicability of the electrochemical immunosensors as well as the colorimetric immunoassays to the determination of AZAs in shellfish, naturally contaminated mussel ( $M$. edulis) samples were analysed using the different approaches.

First, AZAs in a CRM containing AZA-1-3 (certified concentrations) and other analogues (AZA-410) (non-certified concentrations) were determined by the protein $G$ and avidin-biotin interaction-based colorimetric immunoassays and electrochemical immunosensors (Table 2). The immunoapproaches provided a global quantification relative to AZA-1, which was compared with the sum of all different analogues concentrations provided by the NRC. Correlations of $153.4 \%$ and $152.7 \%$ were achieved between the quantifications provided by the protein $\mathrm{G}$ and avidin-biotin interaction-based colorimetric immunoassays, respectively, and NRC values. 
Similar correlations were also obtained in the quantifications provided by the protein G (153.6 $\%)$ and avidin-biotin (154.8\%) electrochemical immunosensors. These percentages higher than $100 \%$ are not surprising taking into account the different recognition principles between analytical methods. While NRC values are obtained by physicochemical approaches, the immunoapproaches provide responses based on the structural recognition of the toxins by the antibody, which can differ between analogues and the assay configuration [25]. Consequently, the application of cross-reactivity factors (CRFs) to each individual AZA analogue concentration can contribute to better understand the correlation between the different analytical methods. Thus, CRFs that had been previously established in the magnetic bead-based immunoassay [25] -where the same PAb and a similar immunological approach were used-were applied to the individual AZA analogue concentrations provided by the NRC. Following the application of the corresponding CRFs to the certified values, correlations of $101.7 \%$ and $101.3 \%$ were achieved between the quantifications provided by the protein $\mathrm{G}$ and avidin-biotin interaction-based colorimetric immunoassays, respectively, and NRC values. Excellent correlations of $101.9 \%$ and $102.7 \%$ were also obtained in the quantifications provided by the protein $\mathrm{G}$ and avidin-biotin electrochemical immunosensors. The obtained results suggest that the PAb recognise all AZA analogues with the same cross-reactivity as in the magnetic bead-based immunoassay and highlight the potential application of all the developed immunoapproaches to the determination of AZAs in mussel samples.

In Ireland, AZAs have been detected in shellfish above the regulatory limit almost every year since the Irish monitoring program was established in 2001 [33]. Raw mussel samples ( $n=16$ ) from this routine monitoring program containing a wide range of AZAs concentrations were selected for their quantification. From these 16 naturally contaminated samples, 11 had been previously analysed by the magnetic bead-based immunoassay [25]. Five additional mussel samples containing AZA-1, AZA-2 and AZA-3 levels below the established $160 \mu \mathrm{g} / \mathrm{kg}$ regulatory limit were included in the analysis to ensure that the developed immunoassays and immunosensors were able to provide reliable AZA quantifications at low concentrations (Table 1). No differences were observed between the quantifications provided by the protein $G$ and avidin-biotin interaction-based colorimetric immunoassays and electrochemical immunosensors $(P=1.00)$, regardless of whether the values were below $(P=0.851)$ or above $(P=0.953)$ the regulatory level. Standard deviations lower than $10 \%$ were obtained for all samples using both approaches.

Since the PAb used in the development of the immunoapproaches was able to recognise AZA carboxy cogeneres in addition to other AZA analogues, the raw mussel samples analysed were 
heated to perform LC-MS/MS analysis. Heating catalyses the decarboxylation of AZA carboxy congeners (e.g., AZA-17, AZA-19, AZA-21 and AZA-23) that may be present in the samples to AZA-3, AZA-6, AZA-4 and AZA-9 respectively [31, 32]. AZA quantifications provided by the protein G-based colorimetric immunoassay $\left(y=1.489 x+42, R^{2}=0.981\right)$ and electrochemical immunosensor $\left(y=1.604 x+7, R^{2}=0.990\right)$ correlated with those obtained by LC-MS/MS analysis $(p<0.0001)$. The immunoassay $\left(y=1.574 x+18, R^{2}=0.990\right)$ and the immunosensor $\left(y=1.582 x+21, R^{2}=0.977\right)$ developed through the avidin-biotin interaction also provided AZA quantifications in correlation with those achieved by the reference method $(p<0.0001)$ (Table 1 , Figure 5). Without the application of the CRFs, the trend observed in the analysis of the mussel samples from the Irish monitoring program was similar to the trend obtained in the analysis of the CRM, being the quantifications achieved by the immunoapproaches $\sim 1.5$-fold those obtained with the reference method. Following the application of the corresponding CRFs to the individual contents determined by LC-MS/MS, AZA quantifications provided by the protein Gbased immunoassay $\left(y=0.965 x+14, R^{2}=0.999\right)$ and immunosensor $\left(y=1.035 x-19, R^{2}=\right.$ $0.999)$ and the avidin-biotin interaction-based immunoassay $\left(y=1.016 x-6, R^{2}=0.998\right)$ and immunosensor ( $\left.y=1.029 x-10, R^{2}=0.999\right)$ were in excellent agreement with LC-MS/MS values (Table 2, Figure 5), with no significant differences observed between the quantifications achieved by any of the immunoapproaches and LC-MS/MS analysis $(P=1.000)$.

All these results indicate without doubt that the developed immunoassays and immunosensors are reliable tools for the screening and quantification of AZAs, facilitating not only the detection of all the regulated AZAs below the regulatory limit, but also other toxic analogues in a simple, rapid and cost-effective manner. 
Table 2. AZA concentrations ( $\mu \mathrm{g} A Z A / \mathrm{kg}$ mussel) of a mussel certified reference material (CRM) and 16 naturally contaminated mussel tissues from the routine monitoring program in Ireland by LC-MS/MS, the colorimetric immunoassays and the electrochemical immunosensors.

\begin{tabular}{|c|c|c|c|c|c|c|c|c|c|c|c|c|c|c|c|c|}
\hline \multirow{3}{*}{ Samples } & \multicolumn{12}{|c|}{ LC-MS/MS } & \multicolumn{2}{|c|}{ Colorimetric immunoasays } & \multicolumn{2}{|c|}{$\begin{array}{l}\text { Electrochemical } \\
\text { immunosensors }\end{array}$} \\
\hline & AZA- & AZA- & AZA- & AZA- & AZA- & AZA- & AZA- & AZA- & AZA- & AZA- & & AZA-1 equiv. & Protein G & Avidin-biotin & Protein G & Avidin-biotin \\
\hline & 1 & 2 & 3 & 4 & 5 & 6 & 7 & 8 & 9 & 10 & $\angle A Z A S$ & $\begin{array}{c}(2 A \angle A S \\
\text { applying CRFs)* }\end{array}$ & AZA-1 equiv. & AZA-1 equiv. & AZA-1 equiv. & AZA-1 equiv. \\
\hline CRM & 1160 & 273 & 211 & 170 & 40 & 90 & 20 & 30 & 40 & 20 & 2054 & 3096 & 3150 & 3137 & 3155 & 3180 \\
\hline Monitoring & & & & & & & & & & & & & & & & \\
\hline$S \# 1$ & 24 & 5 & 15 & nd & nd & 3 & nd & nd & nd & nd & 47 & 77 & 77 & 82 & 79 & 66 \\
\hline S\#2 & 22 & 8 & 15 & nd & nd & 3 & nd & nd & nd & nd & 48 & 77 & 79 & 73 & 80 & 76 \\
\hline S\#3 & 28 & 8 & 17 & nd & nd & 2 & nd & nd & nd & nd & 55 & 86 & 94 & 89 & 82 & 86 \\
\hline SH4 & 21 & 8 & 23 & nd & nd & 3 & nd & nd & nd & nd & 55 & 98 & 95 & 97 & 113 & 65 \\
\hline S\#5 & 33 & 10 & 19 & nd & nd & 2 & nd & nd & nd & nd & 64 & 98 & 105 & 103 & 104 & 100 \\
\hline S\#6 & 49 & 19 & 31 & nd & 1 & 7 & nd & 2 & nd & nd & 109 & 172 & 174 & 177 & 146 & 171 \\
\hline S\#7 & 55 & 19 & 29 & 1 & 1 & 4 & nd & nd & nd & nd & 109 & 165 & 171 & 124 & 159 & 165 \\
\hline S\#8 & 53 & 20 & 31 & 1 & 2 & 4 & nd & nd & nd & nd & 111 & 170 & 133 & 158 & 171 & 130 \\
\hline S\#9 & 128 & 45 & 66 & 3 & 4 & 10 & nd & 5 & nd & nd & 261 & 396 & 412 & 394 & 376 & 414 \\
\hline S\#10 & 148 & 35 & 55 & 3 & 6 & 16 & nd & 1 & 1 & 3 & 268 & 399 & 381 & 391 & 398 & 404 \\
\hline S\#11 & 144 & 59 & 80 & 13 & 5 & 12 & nd & 6 & 1 & 2 & 322 & 515 & 523 & 535 & 513 & 475 \\
\hline S\#12 & 370 & 125 & 216 & 81 & 23 & 44 & 2 & 21 & 19 & 6 & 907 & 1623 & 1667 & 1534 & 1577 & 1646 \\
\hline S\#13 & 425 & 139 & 189 & 77 & 17 & 35 & 4 & 21 & 16 & 4 & 927 & 1558 & 1561 & 1582 & 1550 & 1676 \\
\hline S\#14 & 573 & 164 & 174 & 71 & 13 & 37 & 2 & 20 & 17 & 3 & 1074 & 1656 & 1631 & 1784 & 1661 & 1694 \\
\hline S\#15 & 798 & 258 & 480 & 142 & 32 & 101 & 2 & 46 & 32 & 7 & 1898 & 3356 & 3202 & 3301 & 3399 & 3488 \\
\hline S\#16 & 1524 & 414 & 309 & 132 & 33 & 64 & 10 & 49 & 38 & 9 & 2582 & 3639 & 3509 & 3778 & 3872 & 3664 \\
\hline
\end{tabular}

*Applied CRFs are: AZA-1 = 1; AZA-2 = 0.76; AZA-3 = 2.73; AZA-4 = 3.83; AZA-5 = 1.39; AZA-6 = 2.70; AZA-7 = 2.00; AZA-8 = 1.85; AZA-9 = 2.69; AZA-10 = 2.17 [25] 

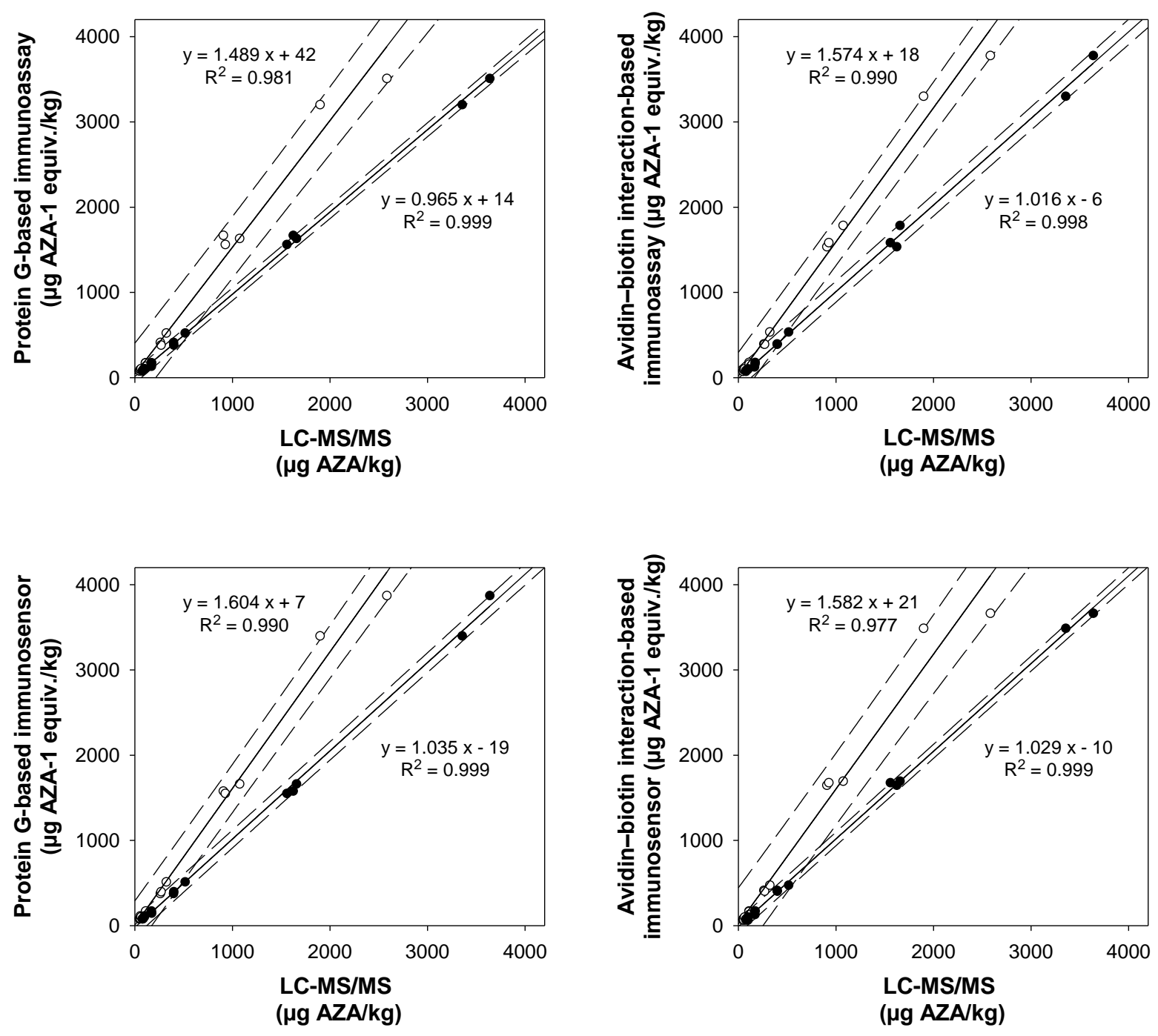

Figure 5. Linear regressions for the correlations between sample quantifications by the colorimetric immunoassays or electrochemical immunosensors and the sum of AZA-1-10 analogues determined by LC-MS/MS analysis, before (white) and after (black) the application of the cross-reactivity factors (CRFs). Dashed lines represent the prediction intervals of $95 \%$. 


\section{CONCLUSIONS}

The immobilisation of the anti-AZA PAb on protein $\mathrm{G}$ or avidin-coated supports via bioaffinity interactions has been used to develop immunoassays and immunosensors with excellent analytical performance, thanks to a controlled and stable antibody immobilisation. All approaches showed a broad working range that enabled the quantification of the current regulated AZAs below the regulatory threshold, but also a broad range of other toxic AZA analogues. No matrix effects were observed and no evaporation of the sample extracts was required, resulting in accurate quantifications with simple and rapid protocols. Although both colorimetric immunoassays and electrochemical immunosensors have been demonstrated to be powerful analytical methods for the reliable determination of AZAs, electrochemical immunosensors provide compact and miniaturised devices that pave the way towards the development of portable tools for in situ measurements. Moreover, the possibility to use lower amounts of immunoreagents, as well as the feasibility to reuse the avidin-biotin interactionbased immunosensors provide clear advantages in terms of sustainability and costeffectiveness.

The good results obtained in the analysis of a considerable amount of naturally contaminated mussel samples evidence that electrochemical immunosensors for the detection of AZAs can be effectively implemented as screening tools in routine monitoring programs, as they provide easy-to-handle, rapid and low-cost high-throughput systems for the specific detection of AZAs in complex matrices. For their practical application in current monitoring programs, it is important to keep in mind that the regulatory limit of $160 \mu \mathrm{g}$ AZA-1 equivalents/kg shellfish established by the European Commission only considers AZA-1-3 (including their toxic potential). As a consequence, in samples where more than these three analogues are present, immunoapproaches could provide results that may lead to a closure of a shellfish harvesting area, while LC-MS/MS analysis could not. This fact is not detrimental for immunoapproaches, but helps to better protect consumer health. Taking this in mind, we propose to establish a "positive", "negative" but also a "suspicious" range to classify samples according to their AZA content. In the case of a "positive" result, a preventive closure of the shellfish harvesting area will be recommended to protect the consumer health and the sample will be analysed by LCMS/MS to confirm the result. In the case of a "suspicious" sample, analysis by LC-MS/MS will help to determine the decision to undertake. By combining screening and confirmatory methods a faster and cost-effective system for marine toxin control in shellfish will be clearly achieved. 


\section{Acknowledgments}

The research leading to these results has received funding under the Sea Change strategy with the support of the Marine Institute and the Marine Research Sub-Programme of the National Development Plan 2007-2013, co-financed under the European Regional Development Fund (ASTOX 2, (PBA/AF/08/001(01)). The publication reflects the views only of the author, and the European Union cannot be held responsible for any use that may be made of the information contained therein. The authors acknowledge financial support from CERCA Programme / Generalitat de Catalunya and Laura Ibañez for her help in the experimental work. Sandra Leonardo acknowledges scholarship from IRTA-Universitat Rovira i Virgili-Banco Santander (2013PIPF URV-IRTA-BS-01).

\section{References}

[1] T. McMahon, J. Silke, Winter toxicity of unknown aetiology in mussels, Harmful Algae News, 14(1996).

[2] V.L. Trainer, L. Moore, B.D. Bill, N.G. Adams, N. Harrington, J. Borchert, et al., Diarrhetic shellfish toxins and other lipophilic toxins of human health concern in Washington State, Marine Drugs, 11(2013) 1815-35.

[3] J. Yao, Z. Tan, D. Zhou, M. Guo, L. Xing, S. Yang, Determination of azaspiracid-1 in shellfishes by liquid chromatography with tandem mass spectrometry, Chinese journal of chromatography, 28(2010) 363-7.

[4] R. Ueoka, A. Ito, M. Izumikawa, S. Maeda, M. Takagi, K. Shin-Ya, et al., Isolation of azaspiracid2 from a marine sponge Echinoclathria sp as a potent cytotoxin, Toxicon, 53(2009) 680-4.

[5] G. Alvarez, E. Uribe, P. Avalos, C. Marino, J. Blanco, First identification of azaspiracid and spirolides in Mesodesma donacium and Mulinia edulis from Northern Chile, Toxicon, 55(2010) 638-41.

[6] A. Lopez-Rivera, K. O'Callaghan, M. Moriarty, D. O'Driscoll, B. Hamilton, M. Lehane, et al., First evidence of azaspiracids (AZAs): A family of lipophilic polyether marine toxins in scallops (Argopecten purpuratus) and mussels (Mytilus chilensis) collected in two regions of Chile, Toxicon, 55(2010) 692-701.

[7] A.D. Turner, A.B. Goya, Occurrence and profiles of lipophilic toxins in shellfish harvested from Argentina, Toxicon, 102(2015) 32-42.

[8] K.J. James, A. Furey, M. Lehane, H. Ramstad, T. Aune, P. Hovgaard, et al., First evidence of an extensive northern European distribution of azaspiracid poisoning (AZP) toxins in shellfish, Toxicon, 40(2002) 909-15.

[9] T. Torgensen, N.B. Bremnes, T. Rundberget, T. Aune, Structural confirmation and occurrence of azaspiracids in Scandinavian brown crabs (Cancer pagurus), Toxicon, 51(2008) 93-101.

[10] B. Krock, U. Tillmann, T.J. Alpermann, D. Voss, O. Zielinski, A.D. Cembella, Phycotoxin composition and distribution in plankton fractions from the German Bight and western Danish coast, Journal of Plankton Research, 35(2013) 1093-108.

[11] Z. Amzil, M. Sibat, F. Royer, V. Savar, First report on azaspiracid and yessotoxin groups detection in French shellfish, Toxicon, 52(2008) 39-48.

[12] P. Vale, R. Bire, P. Hess, Confirmation by LC-MS/MS of azaspiracids in shellfish from the Portuguese north-western coast, Toxicon, 51(2008) 1449-56. 
[13] A.B. Magdalena, M. Lehane, S. Krys, M.L. Fernandez, A. Furey, K.J. James, The first identification of azaspiracids in shellfish from France and Spain, Toxicon, 42(2003) 105-8.

[14] U. Tillmann, D. Jaen, L. Fernandez, M. Gottschling, M. Witt, J. Blanco, et al., Amphidoma languida (Amphidomatacea, Dinophyceae) with a novel azaspiracid toxin profile identified as the cause of molluscan contamination at the Atlantic coast of southern Spain, Harmful Algae, 62(2017) 113-26.

[15] J. Blanco, F. Arevalo, A. Morono, J. Correa, S. Muniz, C. Marino, et al., Presence of azaspiracids in bivalve molluscs from Northern Spain, Toxicon, 137(2017) 135-43.

[16] H. Taleb, P. Vale, R. Amanhir, A. Benhadouch, R. Sagou, A. Chafik, First detection of azaspiracids in mussels in north west Africa, Journal of Shellfish Research, 25(2006) 1067-70.

[17] R. Rossi, C. Dell'Aversano, B. Kcrock, P. Ciminiello, I. Percopo, U. Tillmann, et al., Mediterranean Azadinium dexteroporum (Dinophyceae) produces six novel azaspiracids and azaspiracid-35: a structural study by a multiplatform mass spectrometry approach, Analytical and Bioanalytical Chemistry, 409(2017) 1121-34.

[18] P. Hess, P. McCarron, B. Krock, J. Kilcoyne, C.O. Miles, Azaspiracids: chemistry, biosynthesis, metabolism, and detection, in: L.M. Botana (Ed.), Seafood and Freshwater Toxins. Pharmacology, Physiology, and Detection. CRC Press, Boca Ratom, FL, USA, 2014, pp. 799-822.

[19] European Commission Regulation (EC) No 853/2004 of 29 april 2004 laying down specific hygiene rules for food of animal origin, Official Journal of the European Union L139, 55-205.

[20] S. Leonardo, A. Toldrà, M. Campàs, Trends and prospects on electrochemical biosensors for the detection of marine toxins, in: J. Diogène, M. Campas (Eds.), Recent Advances in the Analysis of Marine Toxins, Comprehensive Analytical Chemistry, 78, 2017, pp. 303-341.

[21] M.O. Frederick, S.D.L. Marin, K.D. Janda, K.C. Nicolaou, T.J. Dickerson, Monoclonal antibodies with orthogonal azaspiracid epitopes, Chembiochem, 10(2009) 1625-9.

[22] C.J. Forsyth, J. Xu, S.T. Nguyen, I.A. Samdal, L.R. Briggs, T. Rundberget, et al., Antibodies with broad specificity to azaspiracids by use of synthetic haptens, Journal of the American Chemical Society, 128(2006) 15114-6.

[23] L.P. Rodriguez, N. Vilarino, M. Carmen Louzao, T.J. Dickerson, K.C. Nicolaou, M.O. Frederick, et al., Microsphere-based immunoassay for the detection of azaspiracids, Analytical Biochemistry, 447(2014) 58-63.

[24] I.A. Samdal, K.E. Lovberg, L.R. Briggs, J. Kilcoyne, J. Xu, C.J. Forsyth, et al., Development of an ELISA for the Detection of Azaspiracids, Journal of Agricultural and Food Chemistry, 63(2015) 7855-61.

[25] S. Leonardo, M. Rambla-Alegre, I.A. Samdal, C.O. Miles, J. Kilcoyne, J. Diogène, et al., Immunorecognition magnetic supports for the development of an electrochemical immunoassay for azaspiracid detection in mussels, Biosensors \& Bioelectronics, 92(2017) 2006.

[26] J. Kilcoyne, M.J. Twiner, P. McCarron, S. Crain, S.D. Giddings, B. Foley, et al., Structure elucidation, relative LC-MS response and in vitro toxicity of azaspiracids 7-10 isolated from mussels (Mytilus edulis), Journal of Agricultural and Food Chemistry, 63(2015) 5083-91.

[27] J. Kilcoyne, A. Keogh, G. Clancy, P. LeBlanc, I. Burton, M.A. Quilliam, et al., Improved isolation procedure for azaspiracids from shellfish, structural elucidation of azaspiracid-6, and stability studies, Journal of Agricultural and Food Chemistry, 60(2012) 2447-55.

[28] G.T. Hermanson, Bioconjugate Techniques, Academic Press, London, 2008.

[29] A. Gerssen, P.P.J. Mulder, M.A. McElhinney, J. de Boer, Liquid chromatography-tandem mass spectrometry method for the detection of marine lipophilic toxins under alkaline conditions, Journal of Chromatography A, 1216(2009) 1421-30.

[30] M. Garcia-Altares, J. Diogene, P. de la Iglesia, The implementation of liquid chromatography tandem mass spectrometry for the official control of lipophilic toxins in seafood: Singlelaboratory validation under four chromatographic conditions, Journal of Chromatography $A$, 1275(2013) 48-60. 
[31] P. McCarron, J. Kilcoyne, C.O. Miles, P. Hess, Formation of azaspiracids-3,-4,-6, and-9 via decarboxylation of carboxyazaspiracid metabolites from shellfish, Journal of Agricultural and Food Chemistry, 57(2009) 160-9.

[32] J. Kilcoyne, P. McCarron, P. Hess, C.O. Miles, Effects of heating on proportions of azaspiracids 1-10 in mussels (Mytilus edulis) and identification of carboxylated precursors for azaspiracids 5, 10, 13, and 15, Journal of Agricultural and Food Chemistry, 63(2015) 10980-7.

[33] Marine Institute Ireland, Latest shellfish safety data. http://www.marine.ie/Home/sitearea/data-services/interactive-maps/latest-shellfish-safety-data/, 2017 (accessed 17.11.17). 